\title{
Rol de los medios de información digitales en los precios en el mercado del arte
}

\section{Por Elena De-la-Poza-Plaza, Natividad Guadalajara-Olmeda e Ismael Moya-Clemente}

\begin{abstract}
Resumen: Se mide la relación entre los medios de información digitales (Google, Yahoo y Enciclopedia Británica) y las ventas en las principales casas de subastas internacionales de obras de arte. Se analiza la capacidad de explicación que las ventas realizadas en el mercado del arte durante los años 1997 2006 tienen sobre las fuentes de información seleccionadas en enero de 2007, observándose una mayor influencia en la Red que en las fuentes enciclopédicas, y mayor en Google que en Yahoo. Se pone de manifiesto la relación, únicamente, entre los medios de información en la Red y las ventas futuras en las casas de subastas a lo largo del año 2007 y también mayor en Google que en Yahoo.
\end{abstract}

Palabras clave: Medios de información digital, Red, Google, Yahoo, Enciclopedia, Obras de arte, Casas de subastas internacionales, Ventas.

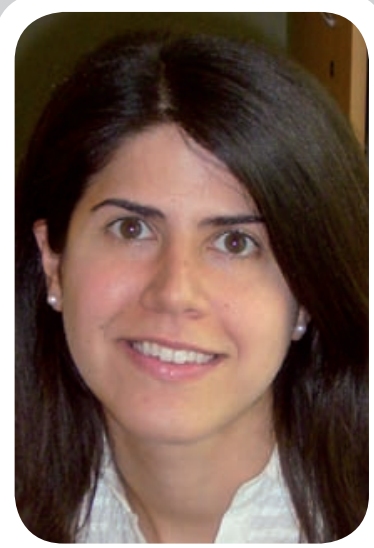

Elena De-la-Poza-Plaza, licenciada en administración y dirección de empresas y doctora en economía por la Universidad Politécnica de Valencia, es profesora ayudante en la misma universidad. Ha realizado estancias predoctorales en Estados Unidos e Italia y ha participado en congresos internacionales en México y Colombia entre otros. Ha publicado artículos sobre valoración de arte y fuentes de información, y un capítulo en Business research yearbook, International Academy of Business Disciplines (IABD).

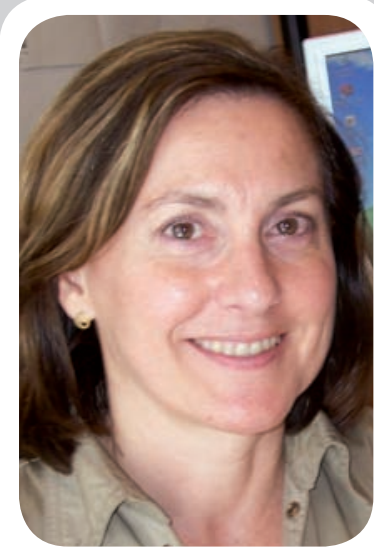

Natividad Guadalajara-OImeda, doctora en ingeniería por la Universidad Politécnica de Valencia, ha impartido docencia en la Facultad de Informática, en la licenciatura de documentación y en la actualidad es profesora de la Facultad de Administración y Dirección de Empresas, de la misma universidad. Dirige un máster de valoración y tasación en sus distintas especialidades: inmobiliaria, obras de arte y empresas. Ha escrito diversos trabajos sobre valoración de obras de arte y sobre documentación.

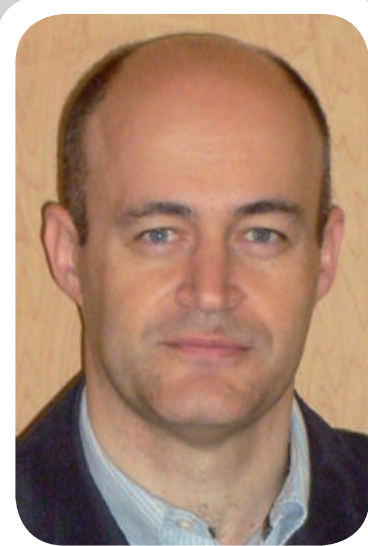

Ismael Moya-Clemente es catedrático de universidad de economía financiera y contabilidad y en la actualidad decano de la Facultad de Administración y Dirección de Empresas de la Universidad Politécnica de Valencia. Ha publicado varios trabajos relacionados con internet, ha presentado una ponencia sobre el mercado del arte en la 15th Intl Conf of Cultural Economics en Boston y ha publicado un capítulo en Business research yearbook, International Academy of Business Disciplines (IABD).

\section{Title: The role of the digital information sources in the art market prices}

Abstract: The objective of this study is to measure the relationship between the digital information sources (Google, Yahoo and Encyclopaedia Britannica) and the turnover of the international art auction houses. The effect of the art turnover (estimated during the period 1997-2006) on the information sources (estimated on January 2007) was analyzed. The results showed a major influence of turnovers on the Net (mainly Google than Yahoo) than on the Encyclopaedia sources. Also, the information sources on the Net only affect the future art auction house's turnovers (Google is more useful than Yahoo).

Keywords: Digital information sources, Net, Google, Yahoo, Encyclopaedia, Art, International auction houses, Turnover.

De-la-Poza-Plaza, Elena; Guadalajara-Olmeda, Natividad; Moya-Clemente, Ismael. "Rol de los medios de información digitales en los precios en el mercado del arte". El profesional de la información, 2009, julio-agosto, v. 18, n. 4, pp. 382-388.

DOI: $10.3145 /$ epi.2009.jul.04

\section{Introducción}

La actual era de la información ha dado lugar a una nueva economía del conocimiento, en la cual juegan un papel primordial los medios de información digitales, especialmente en internet, cuyo uso ha experimentado un crecimiento exponencial en los últimos años. Ya no se puede hablar de un perfil de usuario de internet, puesto que su alcance es universal y se emplea como medio de información principal en todos los sectores 
de la actividad económica: salud, educación, bursátil, inmobiliario, prensa (Orduña-Malea, Ontalba-Ruipérez, 2008; Salaverría, 2005).

La sociedad asiste hoy a un constante cambio, y por ello la Red adquiere una doble función, ya no sólo como medio de información y comunicación, sino también como soporte de las transacciones del mercado (Choi et al., 2002; Weltevreden; Van Rietbergen, 2007; Zentner, 2008) gracias a su rapidez de adaptación y actualización de la información.

Asimismo el análisis de la información en internet (cibermetría) se convierte hoy en objeto de estudio, destacando entre otros el trabajo de Espadas et al. (2008), en el que se analiza el impacto que tiene el correcto posicionamiento de un sitio web en los motores de búsqueda sobre el tráfico de visitas recibidas. Vaughan (2008) determina asimismo la correlación existente entre el número de visitas que reciben los sitios web de las empresas y su rentabilidad.

También en el mercado del arte todos los agentes que intervienen hacen uso de la doble utilidad de internet como medio de información y comunicación y como soporte de su actividad. Destaca en este sentido el análisis realizado por Cueto et al. (2006) en el que se considera el peso de la cultura española mediante los iconos culturales agrupados en las categorías de arte, cine, letras y música, comparándolos con la presencia de iconos de otras lenguas en los principales buscadores. De este análisis se desprende que Estados Unidos acapara la creación de contenidos, y que la mejor asociación a la imagen de España en el campo de las artes se encuentra con los artistas catalanes Miró, Gaudí o Tàpies. En todo caso se constata la relevancia de internet como medio para potenciar la cultura española.

\section{"En el mercado del arte todos los agentes que intervienen hacen uso de la doble utilidad de internet como medio de información y comunicación y como soporte de su actividad"}

Christie's y Sotheby's, principales casas de subastas internacionales, exponen en internet sus colecciones antes de cada sesión, o incluso llevan a cabo algunas de estas sesiones online (Adam, 2002), y sus compraventas son recogidas en amplias bases de datos digitales, aportando transparencia al mercado del arte (Beaux Arts Magazine, 2007).

Los propios artistas utilizan internet para inspirarse y desarrollar su capacidad creativa (Hansen, 2006), así como para promocionar y vender su obra desde sus propios blogs, desde las redes sociales (MySpace, Facebook, LinkedIn) o desde sitios web como eBay o Craiglist (Grant, 2008).

\section{Objetivo}

Los estudios sobre valoración de obras de arte, tanto en el campo académico como en los medios de comunicación tradicionales (prensa, revistas, etc.) son numerosos. En la mayoría de ellos se obtienen modelos matemáticos que estiman el valor de las obras atendiendo a sus particularidades físicas y artísticas, al lugar de venta de la obra, a la fecha de compra-venta, a la nacionalidad del artista y al número de exposiciones que ha realizado. La reputación del artista resulta difícil de cuantificar, por lo que no se ha incluido en dichos análisis, pero es evidente que influye en el valor de las obras de arte y por tanto en el mercado del arte.

Recientemente Caballer y De-la-Poza (2005), y Guadalajara y De-la-Poza (2007) abrieron nuevas líneas de investigación incluyendo en los modelos de valoración la reputación del artista, obtenida a partir de los medios de información digitales y documentales, y estudiando la influencia del volumen de negocio y los precios medios de las obras subastadas de cada artista en dichos medios de información.

Partiendo de estos estudios previos, el presente trabajo tiene una finalidad más amplia: analizar la asociación que puede existir entre la información proporcionada por los motores de búsqueda en internet y la enciclopedia digital, y el volumen de ventas o facturación en el mercado del arte, al objeto de que los interesados puedan emplear la información digital para interpretar el mercado.

En particular, se analizará la utilidad de los medios de información digitales para interpretar las ventas pasadas en el mercado del arte o, por el contrario, para estimar las ventas futuras. Al mismo tiempo se pretende estudiar qué motor de búsqueda y qué tipo de información digital (internet o enciclopedia), se encuentra más relacionado con el mercado del arte.

\section{Fuentes de información}

Se utilizan dos tipos de información digital para definir la reputación del artista:

-La obtenida en internet mediante los buscadores Google y Yahoo.

Se cuantifica en un momento de tiempo determinado el número de resultados obtenidos en los dos buscadores en su versión inglesa, procedentes de toda clase de documentos publicados en la Web (blogs, prensa, revistas, etc.) y que se encuentren relacionados con el artista. 


\section{"Se estudia qué tipo de información digital (internet o enciclopedia) se encuentra más relacionada con el mercado del arte"}

La selección de esos dos buscadores para recoger la información cibermétrica se ha basado en el volumen de consultas realizadas a lo largo del año 2006 por los usuarios de internet en todo el mundo. En ese año, el $60 \%$ del total de las consultas realizadas tuvo lugar desde el buscador Google, seguido de Yahoo, con el 14\% del total. A lo largo del año 2007, ambos mantuvieron sus posiciones en el mercado.

-La información procedente de la Enciclopedia Británica en soporte digital, que se cuantifica por el número de palabras destinadas a cada artista en la obra.

Se ha elegido esta enciclopedia frente a otras también muy importantes y de diferentes nacionalidades, tales como la Universal Larousse Multimedia, la Espasa, la Universal Magna y la Enciclopedia de Arte, porque en un trabajo anterior (Caballer; De-la-Poza, 2005) fue la que mayor relación presentó con la facturación alcanzada por los artistas en las casas de subastas, y por encontrarse en formato digital. Es de esperar que una versión digital tenga mayor capacidad para actualizar la información que otra en formato papel.

Como fuente de información del mercado del arte se ha utilizado la base de datos ArtPrice, que recoge los precios de remate alcanzados por el conjunto de obras subastadas en el mercado internacional del arte, es decir, en el conjunto de casas de subastas mundiales desde el año 1997. La elección de ArtPrice frente a otras bases de datos similares se debe a la extensa información que ésta aporta sobre cada obra y artista, así como a la rápida actualización de la información, y fundamentalmente por su posibilidad de consulta online.

\section{Recogida de datos}

La recogida de la información se ha realizado en varias etapas:

$\mathbf{1}^{\text {a }}$ etapa. Se recopilaron los datos sobre compraventa de obras de arte en casas de subastas a escala internacional contenidos en ArtPrice, estableciendo así una clasificación de los 100 artistas con mayor facturación en el decenio 1997-2006.

Desde el año 2002 hasta la actualidad, la propia ArtPrice publica en el mes de marzo de cada año un informe sobre el mercado del año anterior, en el que se incluye el ranking de los 100 primeros artistas según la facturación alcanzada.
En los años anteriores (1997-2001) esa información no está publicada y para obtenerla ha sido preciso recopilar los precios de remate, recogidos en la base de datos de ArtPrice, alcanzados en las principales casas de subastas internacionales (Christie's, Sotheby's, Ansorena, Durán, etc.) para posteriormente agruparlos por artistas y ordenarlos jerárquicamente, identificando así los 100 primeros de cada año.

Se estima que 10 años es un período de tiempo suficientemente amplio para hacer una selección de los artistas con mayor presencia en el mercado y que sean representativos del mismo. También un decenio recoge años de bonanza económica y otros de recesión, a fin de aislar el posible efecto que pudieran tener los aspectos macroeconómicos. Igualmente puede haber años en los que, por conmemorarse la muerte o nacimiento de un artista, o por la celebración de multitud de exposiciones $o$ actos conmemorativos de obras de arte en todo el mundo, se distorsione el volumen de ventas de sus obras.

El empleo de un período de tiempo más amplio, aunque sería deseable, no es posible debido a la disponibilidad limitada de la información sobre la facturación en ArtPrice.

Aunque en un principio se estudiaron los 100 primeros artistas, se observó que operativamente es difícil trabajar con un número tan elevado, ya que el ranking de cada artista varía de un año a otro y cada año cambia la composición de la muestra, excepto para el caso de Picasso que siempre ocupó el primer puesto en todo el período considerado. Con el fin de mantener constante la muestra de artistas analizados, se llevó a cabo el siguiente proceso de selección:

- De los 100 artistas se seleccionaron únicamente los 40 primeros, ya que éstos constituyen un porcentaje de las ventas en el mercado del arte muy elevado y, por ello, la tendencia del mismo se ve afectada en gran medida por su evolución.

- De esta primera selección se identificaron aquellos artistas que tuvieron ventas todos los años del decenio, con la doble finalidad de elegir figuras de gran peso en el mercado y con una presencia continua en el mismo. De esta manera la muestra quedó reducida a 22 personas.

En la tabla 1 se recoge la relación de artistas y su facturación total en dichos años, considerando todas las disciplinas artísticas, como escultura, pintura, grabado, fotografía, dibujo, acuarela, cerámica, etc., y cada una de ellas en sus diversas variantes en cuanto a soportes se refiere.

$2^{\text {a }}$ etapa. La elaboración de las variables cibermétricas se realizó en enero de 2007. El tipo de búsqueda se ha llevado a cabo de dos formas diferentes: una, 
introduciendo exclusivamente el apellido del artista (cibermétrica-sin) y otra, considerando el nombre y apellidos del mismo y su fecha de nacimiento y muerte (cibermétrica-con). Con este segundo tipo de búsqueda lo que se pretende es eliminar las citas que no estén relacionadas directamente con el artista. De esta forma, para cada artista se obtuvieron 4 datos o información cibermétrica y un dato enciclopédico, que recoge el número de palabras escritas sobre cada artista en la edición online de la Enciclopedia Británica en enero de 2007 (tabla 1).

\section{"Los resultados en los buscadores y en la enciclopedia digital están relacionados con la facturación que ha tenido lugar en el mercado del arte"}

$3^{\mathbf{a}}$ etapa. En 2008 se obtuvo, también de la base de datos ArtPrice, el volumen de facturación alcanzado a lo largo del año 2007 para cada uno de los artistas (véase columna 3 de la tabla 1).

\section{Metodología y resultados}

Se contrastan dos hipótesis:

1. Los resultados en los buscadores (Google y Yahoo) y en la enciclopedia digital (número de palabras) están relacionados con la facturación que ha tenido lugar en el mercado del arte.

2. El volumen de ventas en el mercado del arte a corto plazo está relacionado con los resultados en los buscadores (Google y Yahoo) y en la enciclopedia digital (número de palabras).

La metodología empleada en el contraste de las hipótesis propuestas ha sido el análisis de regresión sim-

\begin{tabular}{|c|c|c|c|c|c|c|c|}
\hline \multirow[b]{2}{*}{ Artista } & \multicolumn{2}{|c|}{$\begin{array}{l}\text { Facturación total } \\
\qquad(\text { miles } €)\end{array}$} & \multicolumn{2}{|c|}{$\begin{array}{c}\text { Variable } \\
\text { cibermétrica-sin } \\
\text { (miles de } \\
\text { resultados) }\end{array}$} & \multicolumn{2}{|c|}{$\begin{array}{c}\text { Variable } \\
\text { cibermétrica-con } \\
\text { (miles de } \\
\text { resultados) }\end{array}$} & \multirow{2}{*}{$\begin{array}{c}\begin{array}{c}\text { Variable } \\
\text { enciclopedia } \\
\text { (no palabras) }\end{array} \\
\begin{array}{c}\text { Británica } \\
\text { online }\end{array} \\
\end{array}$} \\
\hline & 1996-2006 & 2007 & Google & Yahoo & Google & Yahoo & \\
\hline Picasso, Pablo & 1.535 .089 & 230.181 & 31.000 & 16.500 & 2.660 & 9.520 & 5.811 \\
\hline Warhol, Andy & 897.317 & 304.020 & 11.000 & 6.040 & 2.670 & 11.900 & 440 \\
\hline Monet, Claude & 640.497 & 118.748 & 16.000 & 9.710 & 2.040 & 5.100 & 2.942 \\
\hline Kooning, Willem de & 405.936 & 51.640 & 1.080 & 515 & 759 & 326 & 1.315 \\
\hline Chagall, Marc & 364.852 & 63.802 & 4.740 & 2.150 & 942 & 327 & 1.932 \\
\hline Basquiat, J ean-Michel & 299.211 & 73.085 & 1.750 & 735 & 117 & 54 & 558 \\
\hline Léger, Fernand & 287.339 & 66.444 & 18.600 & 240 & 1.400 & 5.610 & 1.106 \\
\hline Lichtenstein, Roy & 263.928 & 47.213 & 8.900 & 1.650 & 1.370 & 681 & 100 \\
\hline Miró, Joan & 237.052 & 41.351 & 22.900 & 1.880 & 2.090 & 506 & 393 \\
\hline Matisse, Henri & 219.554 & 60.582 & 7.670 & 3.640 & 1.800 & 3.180 & 1.363 \\
\hline Renoir, Auguste & 230.241 & 82.214 & 7.130 & 3.370 & 1.610 & 2.080 & 2.711 \\
\hline Dongen, Kees van & 159.834 & 61.250 & 1.780 & 1.200 & 561 & 153 & 1.978 \\
\hline Giacometti, Alberto & 166.232 & 22.198 & 2.090 & 736 & 1.030 & 832 & 267 \\
\hline Modigliani, Amedeo & 208.618 & 61.954 & 3.610 & 1.410 & 1.020 & 758 & 1.135 \\
\hline Degas, Edgar & 153.754 & 52.703 & 4.970 & 2.100 & 1.660 & 2.010 & 1.012 \\
\hline Pissarro, Camille & 146.791 & 18.002 & 1.270 & 595 & 1.220 & 592 & 4.359 \\
\hline Calder, Alexander & 192.047 & 41.863 & 8.220 & 4.560 & 1.310 & 2.230 & 2.639 \\
\hline Fontana, Lucio & 188.876 & 22.444 & 23.300 & 1.230 & 1.030 & 12.400 & 1.380 \\
\hline Cézanne, Paul & 166.435 & 62.339 & 4.450 & 2.970 & 3.860 & 3.370 & 3.112 \\
\hline Richter, Gerhard & 166.985 & 61.835 & 44.800 & 24.200 & 1.210 & 926 & 455 \\
\hline Twombly, Cy & 109.395 & 9.368 & 356 & 131 & 793 & 427 & - \\
\hline Vlaminck, Maurice de & 113.629 & 25.943 & 542 & 177 & 338 & 86 & 300 \\
\hline
\end{tabular}

Tabla 1. Ranking de artistas por volumen de ventas y número de resultados en los motores de búsqueda en internet y número de palabras en la enciclopedia. 
ple. El objeto de este tipo de análisis es investigar o explicar la relación estadística que hay entre una variable dependiente [en este caso la facturación en el mercado del arte (columnas 2 y 3 de la tabla 1)], y otra variable independiente [(variables cibermétricas: número de resultados en los motores de búsqueda Google y Yahoo; o variable enciclopedia digital: número de palabras) (columnas 4 a 8 de la tabla 1)]. Como medida de bondad del ajuste lineal se utiliza el coeficiente de determinación, que mide la proporción de variabilidad total de la variable dependiente, esto es, la capacidad explicativa del modelo.

\subsection{Primera hipótesis}

En los gráficos 1, 2 y 3 , se recogen los pares de valores y los modelos de regresión.

Se observa cómo cuanto mayor es el número de resultados de los buscadores, mayor es la facturación pasada y además el tipo de relación que se establece es lineal o proporcional.

Económicamente el efecto se cuantifica del siguiente modo: la facturación pasada en el período 1997-2006 aumenta de forma constante en 44,28 y 43,65 euros por cada resultado adicional que consideren los buscadores Google y Yahoo para cada artista respectivamente.

Además los valores obtenidos con el buscador Yahoo varían mucho más, respecto a la recta de ajuste,

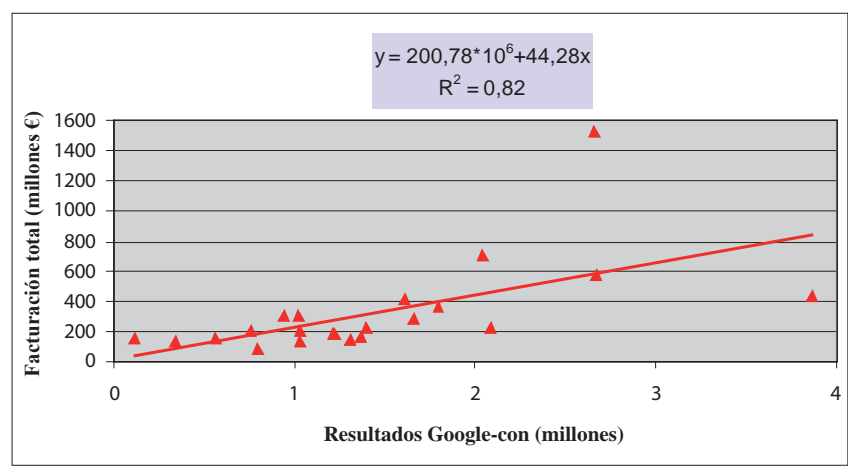

Gráfico 1. Estimación del modelo de regresión para el período 1997. 2006 de la facturación total respecto de la información cibermétrica Google-con, en enero de 2007

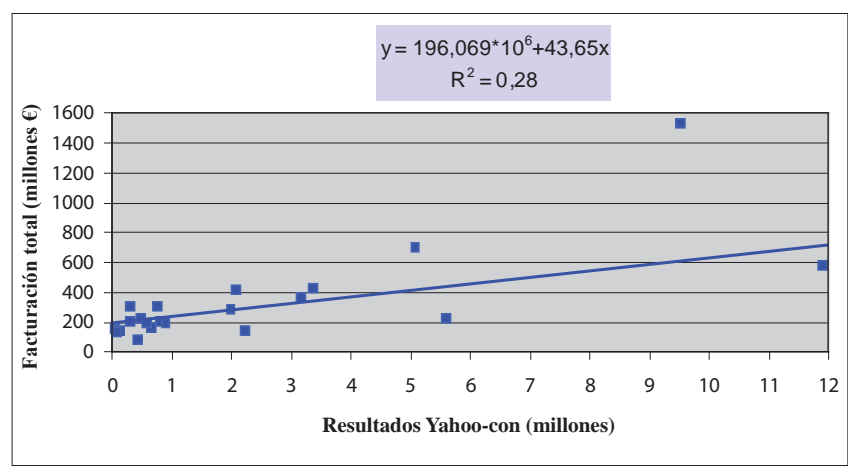

Gráfico 2. Estimación del modelo de regresión para el período 1997. 2006 de la facturación total respecto de la información cibermétrica Yahoo-con, en enero de 2007

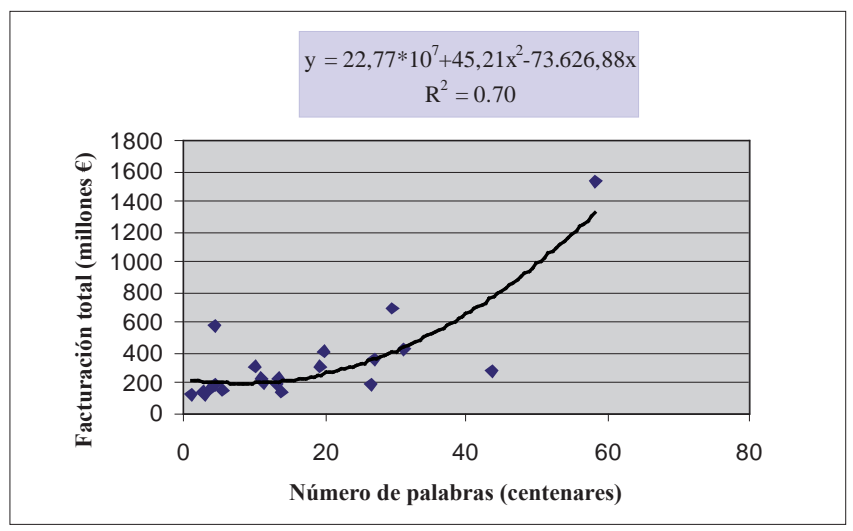

Gráfico 3. Estimación del modelo de regresión para el período 1997. 2006 de la facturación total respecto de la información digital de la Enciclopedia Británica online en enero de 2007

que los obtenidos con Google, lo que indica la preferencia de Google respecto a Yahoo, y queda confirmado estadísticamente con una bondad del ajuste del $82 \%$ en Google y del 28\% con Yahoo.

Los resultados cibermétrica-sin no son significativos en el análisis, lo que ratifica la necesidad de especificar claramente el nombre y las fechas de cada artista en las búsquedas.

El gráfico 3 muestra cómo, en general, a mayor facturación, mayor es el número de palabras recogidas en la enciclopedia, pero no de forma lineal, sino creciente, de manera que a medida que aumenta el número de palabras, el incremento de las ventas pasadas es mucho mayor.

La variabilidad explicada entre la facturación y la variable Enciclopedia Británica en formato digital es del 70\%, inferior a la obtenida con Google, pero mucho mayor que con Yahoo.

\section{"A mayor facturación, mayor es el número de palabras recogidas en la enciclopedia"}

\subsection{Segunda hipótesis}

Para su verificación se analiza ahora la relación entre la facturación en todo el año 2007 y la misma información cibermétrica y en la enciclopedia en el mes de enero de 2007, la cual se recoge en los gráficos 4, 5 y 6.

Los resultados en los buscadores Google y Yahoo permiten estimar también las ventas a corto plazo de manera lineal en 6,35 y 12,67 euros por cada resultado adicional en Google y Yahoo, respectivamente.

De nuevo el buscador Google presenta un mayor poder explicativo que Yahoo, del $77 \%$ y $49 \%$ respectivamente. 


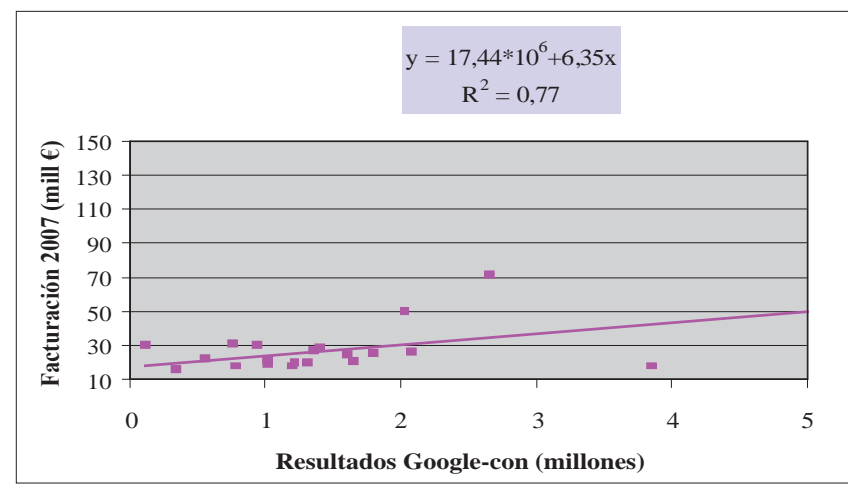

Gráfico 4. Estimación del modelo de regresión de la facturación en el año 2007 respecto de la información cibermétrica Google-con, en enero de 2007

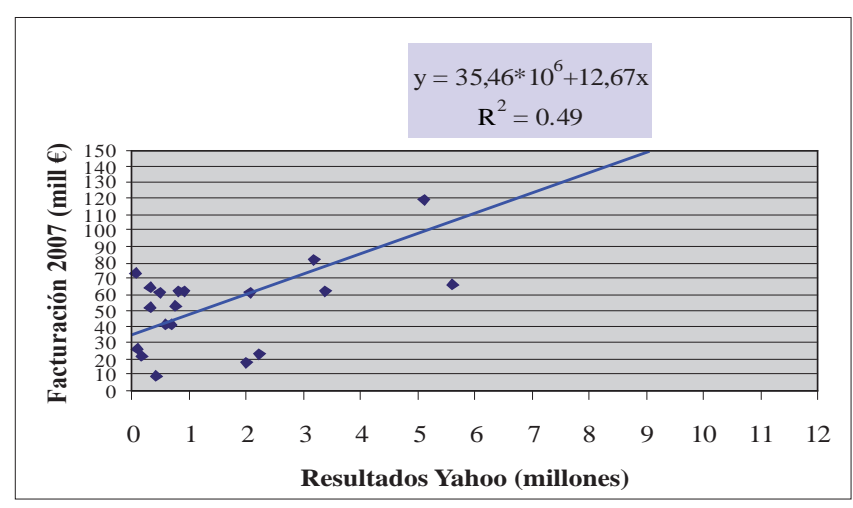

Gráfico 5. Estimación del modelo de regresión de la facturación en el año 2007 respecto de la información cibermétrica Yahoo-con, en enero de 2007

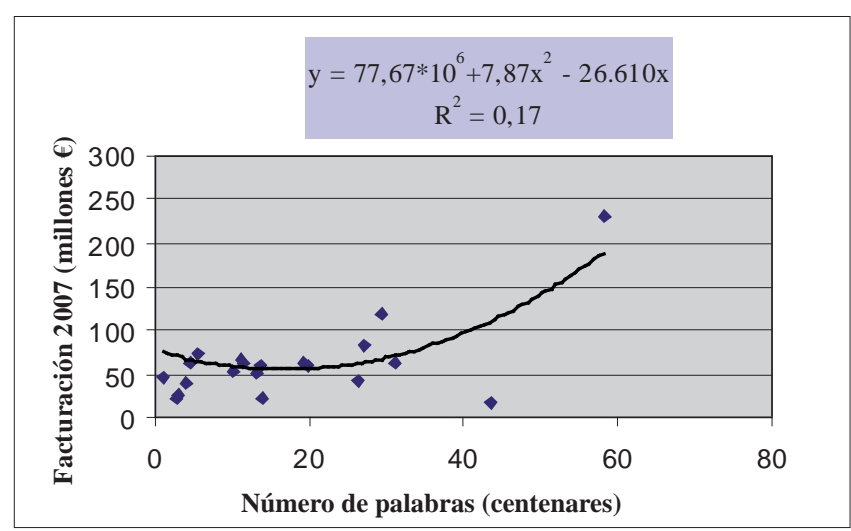

Gráfico 6. Estimación del modelo de regresión de la facturación en el año 2007 respecto de la información en la Enciclopedia Británica online en enero de 2007

También ahora las citas cibermétrica-sin (sólo con el apellido del artista), de la misma forma que ocurrió en la primera hipótesis, no resultaron explicativas desde un punto de vista estadístico.

Análogamente en el gráfico 6 se observa cómo la relación entre la facturación anual del 2007 y la información en la enciclopedia es cuadrática, es decir, cada palabra adicional supone un incremento mayor en la facturación futura del artista en el año 2007, si bien la relación entre ambas variables es débil, del 17\% únicamente.

\section{Conclusiones}

Se demuestra que existe una relación entre la información proporcionada por los buscadores en internet y por la enciclopedia, y el volumen de ventas o facturación en el mercado del arte, tanto en el pasado como en el futuro a corto plazo.

No obstante, esta relación entre las ventas en el mercado del arte y la información cibermétrica en el buscador Google y la enciclopedia digital es mayor cuando se trata de ventas pasadas que de futuras, lo que parece indicar que esta información digital es capaz de recoger en mayor grado lo acontecido en el mercado que de estimar a corto plazo las ventas futuras.

Por el contrario, el buscador Yahoo es más útil para explicar las ventas en un futuro próximo que en un amplio período de tiempo pasado.

\section{"Google demuestra un mayor poder explicativo que Yahoo, tanto de las ventas pasadas como de las futuras"}

Si se comparan los motores de búsqueda, Google demuestra un mayor poder explicativo que Yahoo, tanto de las ventas pasadas como de las futuras, mientras que la información en la enciclopedia resulta útil para interpretar las ventas pasadas, pero no para predecir las ventas futuras a corto plazo.

No obstante, sería necesario repetir el análisis en años posteriores y comprobar si realmente se reproducen dichos comportamientos en un período de tiempo más largo.

En resumen, estos resultados abren un camino a nuevas investigaciones que permitan valorar al artista mediante su repercusión en la cibermetría y en la Enciclopedia Británica online, fundamentalmente en la primera, así como emplear los medios de información digitales para analizar el mercado del arte a medio y largo plazo.

\section{Bibliografía}

Adam, Georgina. "The opportunity to find bargains?". Art newspaper, 2002, January, v. 13, n. 121, pp. 33.

"Artprice.com: les raisons d'un succès". Beaux arts magazine, 2007, Juin, n. 276 , pp. $68-69$.

Caballer, Vicente; De-la-Poza, Elena. "Modelos econométricos para la valoración de obras de arte pictóricas". En: VIII Congreso Internacional Cultura Europea. Universidad de Navarra, 2005. pp. 1-10.

Cueto, Luís; Noya, Javier; Soler, Chimo. "El valor de los iconos culturales en internet”. En: Enciclopedia del español en el mundo. Anuario del Instituto Cervantes 2006-2007. 
